\title{
Inverse Problem of Variational Calculus
}

\author{
Pedro Gonçalves Henriques \\ Centro de Análise Matemática, Geometria e Sistemas Dinâmicos \\ Departamento de Matemática, Instituto Superior Técnico \\ Av. Rovisco Pais, 1049-001 Lisboa, Portugal \\ E-mail address: phenr@math.ist.utl.pt
}

\begin{abstract}
We will discuss the so-called mixed endpoint conditions for variational problems with non-holonomic constraints given by form actions of order greater than one. We will present some results and discuss the inverse problem of Calculus of Variations.
\end{abstract}

\section{Introduction}

In the present text we describe new results for the the inverse problem of Variational Calculus for multiple integrals in the context of exterior differential systems. We deal with non-holonomic constraints in the setting of the mixed endpoint conditions defined by P. A. Griffiths ([16]). This work is a follow up of ([29]). In section I and II we present a short review of the latter work. In section III we discuss the inverse problem of Variational Calculus, and conclude in section IV with a study of the Generalized Lagrange Problem with non-holonomic constraints.

Caratheodory [1929], Weil-De Donder [1936] and Lepage [1936-1942] were the first to study Variational Calculus for multiple integrals. Later Dedecker [1953-1977], Liesen [1967], R. Hermann [1966], H. Goldschmidt and S. Sternberg [1973], Ouzilou [1972], D. Krupka [1970-1975], I. M. Anderson [1980], P. L. Garcia and A. Pérez-Rendón [1969-1978], C. Günther [1987], Edelen [1961] and Rund [1966], gave their contributions to this field.

In 1983 Griffiths (see [16]) presented a new approach to variational problems in the context of exterior differential systems. His work in the set of one-dimensional integral manifolds of a differential system $\left(I^{*}, L^{*}\right)$ addressed the problem of finding extrema for the functional $\phi$ using intrinsic

Partially supported by FCT/Portugal through the Center for Mathematical Analysis, Geometry, and Dynamical Systems. 
entities. He established the mixed endpoint conditions for problems with non-holonomic constraints.

The Inverse Problem of Variational Calculus was presented in 1887 by Helmholtz in the following way: given $P_{i}=P_{i}\left(x, u^{j}, u_{x}^{j}, u_{x x}^{j}\right)$ is there a Lagrangian $L\left(x, u^{j}, u_{x}^{j}\right)$ such that $E_{i}(L)=\partial L / \partial u^{i}-D_{x} \partial L / \partial u_{x}^{i}=P_{i}$ where $D_{x}=\partial / \partial x+u_{x}^{i} \partial / \partial u^{i}+u_{x x}^{i} \partial / \partial u_{x}^{i}$ ? He found necessary conditions for $P_{i}$ to be a Euler-Lagrange system (see (3.1) (3.2) and (3.3)). Years later these equations where proved to be locally sufficient conditions.

I. M. Anderson [1992], [1980], P. J. Olver [1986], F. Takens [1979], W. M. Tulczyjew [1980] and A. M. Vinagradov [1964] generalized Helmholtz's conditions both to higher order systems of partial differential equations and to multiple integrals.

1.1. Integral manifolds and valued differential systems. Let $X$ be a manifold and assume that a Pfaffian differential system $\left(I^{*}, L^{*}\right)$ is given by:

i) a subbundle $I^{*} \subset T^{*} X$,

ii) another subbundle $L^{*} \subset T^{*} X$ with $I^{*} \subset L^{*} \subset T^{*} X$,

such that the rank $\left(L^{*} / I^{*}\right)=n$ (with $n$ being a natural number).

An integral manifold of $\left(I^{*}, L^{*}\right)$ is given by an oriented connected compact $n$-dimensional smooth manifold $\mathrm{N}$ (possibly with a piecewise smooth boundary $N$ ) together with a smooth mapping

$$
f: N \rightarrow X
$$

satisfying:

$$
I_{f(x)}^{*} \stackrel{\perp}{=} L_{f(x)}^{*}{ }^{\perp}+f_{*}(T N),
$$

for all $x \in N$.

$V\left(I^{*}, L^{*}\right)$ is the collection of integral manifolds $f$ of $\left(I^{*}, L^{*}\right)$.

A valued differential system is a triple $\left(I^{*}, L^{*}, \varphi\right)$, where $\left(I^{*}, L^{*}\right)$ is a Pfaffian differential system and $\varphi$ is an n-form on $X$.

We define the functional $\phi$ associated with $\left(I^{*}, L^{*}, \varphi\right)$ in $V\left(I^{*}, L^{*}\right)$ by:

$$
\begin{aligned}
& \phi: V\left(I^{*}, L^{*}\right) \rightarrow R, \\
& f \rightarrow \phi[f]=\int f^{*} \varphi .
\end{aligned}
$$


1.2. Local embeddability. Let us assume that

$$
d\left(C^{\infty}\left(X, L^{*}\right)\right) \subset C^{\infty}\left(X, L^{*} \wedge T^{*} X\right)
$$

and let $d^{\prime}=\operatorname{dim} X, \quad s=\operatorname{rank} I^{*},\left(d\left(C^{\infty}\left(X, L^{*}\right)\right)\right.$ is the set of images by the exterior derivative of $\left.C^{\infty}\left(X, L^{*}\right)\right)$. Using the Frobenius theorem, we can set for every $p \in X$ a chart coordinate system $\left\{u^{1}, \ldots, u^{s+n}, v^{1}, \ldots, v^{d^{\prime}-s-n}\right\}$ so that:

i)

$$
\left.L^{*}=\operatorname{span}\left\{d u^{\alpha} \mid 1 \leq \alpha \leq s+n\right\}\right)
$$

ii)

$$
L^{* \perp}=\operatorname{span}\left\{\frac{\partial}{\partial v^{i}} \mid 1 \leq i \leq d^{\prime}-s-n\right\}
$$

for an open subset $U$ of $X$ with $p \in U$.

Definition 1.1. Let $\left(I^{*}, L^{*}\right)$ be a Pfaffian differential system with

$$
d\left(C^{\infty}(X, L)\right) \subset C^{\infty}\left(X, L^{*} \wedge T^{*} X\right) .
$$

$\left(I^{*}, L^{*}\right)$ is locally embeddable if for every $p \in X$ there exists an open neighborhood $U$ of $p$ and local coframes $C F=\left\{\theta_{1}, \ldots, \theta_{s}\right\}$ for $I^{*}$ and $C F^{\prime}=$ $\left\{\theta_{1}, \ldots, \theta_{s}, d u^{\prime s+1}, d u^{\prime s+n}\right\}$ for $L_{U}^{*}$ satisfying the following conditions:

(i) $\left.\delta\left(I_{U}^{*} \wedge \Omega\right) \subset T^{*} U \wedge \wedge^{n}\left(L_{U}^{*}\right) / T^{*} U \wedge I_{U}^{*} \wedge \wedge^{n-1}\left(L^{*}\right)\right)$

(ii) Ker $\delta$ is a constant rank subbundle of $I^{*} \wedge \Omega$,

where $\Omega=\operatorname{span}\{d u " s+1 \wedge \ldots \wedge \widehat{d u " s+\beta} \wedge \ldots \wedge d u " s+n\}, \widehat{d u^{\prime s+\beta}}$ - means deletion of $s+b$ factor (for $n=1, \widehat{d u^{\prime \prime} s+1}=1$ ).

The map $\delta: I^{*} \wedge \Omega \rightarrow \wedge^{n+1}\left(T^{*} U\right) / I_{u}^{*} \wedge\left(\wedge^{n}\left(T^{*} U\right)\right)$ is induced by:

$$
d: C^{\infty}\left(U, I^{*} \wedge \Omega\right) \rightarrow C^{\infty}\left(U, \wedge^{n+1}\left(T^{*} U\right)\right)
$$

in $I^{*} \wedge \Omega$, i.e, if $I^{*}$ has no Cauchy characteristics, the structure equations are locally:

$$
d \theta^{i} \equiv \pi_{j}^{i} \wedge d u^{\prime s+j}+A_{i^{\prime} \alpha}^{i j^{\prime}} \pi_{j^{\prime}}^{i^{\prime}} \wedge \theta^{\alpha}+B_{\alpha \beta}^{i} \theta^{\alpha} \wedge d u^{\prime s+\beta} \bmod I \wedge I
$$

$1 \leq i, i^{\prime}, \alpha \leq s, 1 \leq j, j^{\prime}, \beta \leq n, I=C^{\infty}\left(X, I^{*}\right)$.

In $([26])$ we showed that if $\left(I^{*}, L^{*}\right)$ is locally embeddable, there exist locally defined mappings that induce $\left(I^{*}, L^{*}\right)$ from the canonical system in $J^{1}\left(R^{n}, R^{s}\right)$ with possible constraints, giving a local correspondence between these two differential systems. These sets of systems form a general class that can be described locally by the canonical system of $J^{1}\left(R^{n}, R^{s}\right)$. 
1.3. The Cartan system. Let $\left(I^{*}, L^{*}, \varphi\right)$ be a valued differential system on $X$, and $W$ be the total space of $I^{*}$. Let $\chi$ be the canonical form on $T^{*} X$, and $i$ the inclusion map $W \stackrel{i}{\hookrightarrow} T^{*} X$.

Let us assume that there exists a local $n$-form $\omega$ inducing a nonzero section of $\wedge^{n}\left(L^{*} / I^{*}\right)$ with the following form:

$$
\begin{gathered}
\omega=\omega^{1} \wedge \ldots \wedge \omega^{n} \\
\omega_{i}=(-1)^{i-1} \omega^{1} \wedge \ldots \wedge \widehat{\omega^{i}} \ldots \wedge \omega^{n}
\end{gathered}
$$

Let $W^{n}$ be the $n$-Cartesian power of $W$, and $Z$ be a subset of $W^{n}$ defined by $Z=\left\{z \in W^{n}: \pi^{\prime}(z) \in \Delta X^{n}\right\}$, where $\pi^{\prime}$ is the natural projection $\pi^{\prime}(z): W^{n} \rightarrow X$, and $\Delta X^{n}$ is the diagonal submanifold of $X^{n}$.

The subset $Z$ is a vector subbundle over $X$ and $\operatorname{dim} Z=d+s n$ with $d \leq d^{\prime}$. We define

$$
\Psi=d \psi
$$

where $\psi$ is given by

$$
\psi=\pi^{*} \varphi+\left(\pi^{j} o i^{\prime}\right)^{*}\left[i^{*}(\chi)\right] \wedge \pi^{*} \omega_{j} .
$$

$\pi^{j}$ is the natural projection into the $j^{\text {th }}$ component $\pi^{j}: W^{n} \rightarrow W$, i' is the inclusion map $Z \rightarrow W^{n}$ and $\pi$ is the natural projection $\pi: Z \rightarrow X$. Locally $\left(\pi^{j} o i^{\prime}\right)^{*}\left[i^{*}(\chi)\right] \wedge \pi^{*} \omega_{j}=\lambda_{j}^{i} \theta_{i}{ }^{j}$ with $\theta_{i}{ }^{j}=\theta^{j} \wedge \omega_{j}$.

Definition 1.2. Given the $n+1$-form $\Psi$, the Cartan system $C(\Psi)$ is the ideal generated by the set of $n$-forms $\{v\lrcorner \Psi$ where $\left.v \in C^{\infty}(Z, T Z)\right\}$. An integral manifold of $(C(\Psi), \omega)$ is given by an oriented connected compact $n$ dimensional smooth manifold $N$ (possibly with a piecewise smooth boundary $\partial N)$ together with a smooth mapping $f: N \rightarrow X$ satisfying:

$$
f^{*} \theta=0 \quad \text { for every } \quad \theta \in C(\Psi)
$$

and

$$
f^{*}(\omega) \neq 0
$$

Solutions of $(C(\Psi), \omega)$ projected in $X$ will give a candidate for extremum of $\phi$ with suitable boundary conditions.

$$
\left.\left.\delta \phi=\int_{f(N)} v\right\lrcorner d \psi+d(v\lrcorner \psi\right)
$$


1.4. The momentum space. Suppose we are given on $Z$ :

(i) a closed $(n+1)$-form $\Psi$ with the associated Cartan system $C(\Psi)$,

(ii) $\pi^{\prime *} \omega$ : the pull back to $Z$ of $\omega$, the $n$-form which induces a nonzero section on $\wedge^{n}\left(L^{*} / I^{*}\right)$.

Definition 1.3. Let $\left(C(\Psi), \pi^{\prime *} \omega\right)_{n}$ be the ideal generated by $\left(C(\Psi), \pi^{\prime *} \omega\right)$ in $C^{\infty}\left(Z, \wedge^{n} T^{*} Z\right), z_{0} \in Z$ and $E_{0}^{p}$ a p-dimensional subspace of the tangent space $T_{z_{0}}$. We say that $\left[z_{0}, E_{0}^{p}\right]$ is a p-dimensional integral element of $\left(C(\Psi), \pi^{* *} \omega\right)_{n}$ if

(i) $<E_{0}^{p}, \alpha>=0$ for all $\left(C(\Psi), \pi^{\prime *} \omega\right)_{n}$

(ii) $\left.<E_{0}^{p}\right\lrcorner \omega>\neq 0$

The set of integral elements $\left[z_{0}, E_{0}^{n}\right]$ gives a subset

$$
\left.V_{n}\left(C(\Psi), \pi^{*} \omega\right)\right) \subset G_{n}(Z) .
$$

Denoting by $\pi$ " the projection $G_{n}(Z) \rightarrow Z$ and assuming regularity at each step, one inductively defines:

$$
\begin{gathered}
\left.\left.Z_{1}=\pi "\left(V_{n}\left(C(\Psi), \pi^{*} \omega\right)\right), V_{n}^{\prime}\left(C(\Psi), \pi^{*} \omega\right)\right)\right)= \\
\left\{E \in V_{n}\left(C(\Psi), \pi^{*} \omega\right): E \text { tangent to } Z_{1}\right\} \\
\left.\left.Z_{2}=\pi^{\prime \prime}\left(V_{n}^{\prime}\left(C(\Psi), \pi^{*} \omega\right)\right), V^{\prime \prime}{ }_{n}\left(C(\Psi), \pi^{*} \omega\right)\right)\right)= \\
\left\{E \in V_{n}^{\prime}\left(C\left(\Psi, \pi^{*} \omega\right): E \text { tangent to } Z_{2}\right\} \ldots\right.
\end{gathered}
$$

Definition 1.4. $\left(I^{*}, L^{*}, \varphi\right)$ is a locally embeddable valued differential system, and $\omega=\omega^{1} \wedge \ldots \wedge \omega^{n}$. If there exists a $k_{0} \in N$, such that $Z_{k_{0}}=$ $Z_{k_{0}+1}=\ldots=Z_{k_{0}+n^{\prime}}\left(n^{\prime} \in N\right)$ in the above construction, with:

(i) $Z_{k_{0}}$ being a manifold of dimension $(n+1) m+n$ for $m \in N$, and

(ii) $\left(C(\Psi), \pi^{*} \omega\right) Z_{k_{0}}$ being a differential system in $Z_{k_{0}}$ with $r_{n}=0$ (Cartan number in Cartan-Kähler Theorem) for all $\left.V_{n-1}\left(C(\Psi), \pi^{*} \omega\right)\right)$.

Then $\left(I^{*}, L^{*}, \varphi\right)$ is a non-degenerate valued differential system, and $Z=Y$ is called the momentum space.

For $n=1$ we follow [16] and replace condition (ii) by $\psi \wedge \Psi^{n} \neq 0$ on $Z_{k_{0}}$.

We call $\left(C(\Psi), \pi^{*} \omega\right)_{Y}$ the prolongation of $\left(C(\Psi), \pi^{*} \omega\right)$ in the momentum space. By construction, the differential system $\left(C(\Psi), \pi^{*} \omega\right)_{Y}$ satisfies:

(i) the projection $\left(C(\Psi), \pi^{*} \omega\right) \rightarrow Y$ is surjective,

(ii) the integral manifolds of $\left(C(\Psi), \pi^{* *} \omega\right)$ on $Z$ coincide with those of $\left(C(\Psi), \pi^{*} \omega\right)$ on $Y$. 
1.5. Well-posed valued differential systems. Suppose we have the following subbundles of $T^{*} X$ such that:

(a)

$$
\begin{array}{lllll}
I^{*} & \subset & L^{*} & \subset & T^{*} X \\
P^{*} & \subset & M^{*} & &
\end{array}
$$

(b) the locally given $\mathrm{n}$-form $\omega$ also induces a nonzero section on $\wedge^{n}\left(M^{*} / P^{*}\right)$

(c) $\left.Y \subset\left(P^{*}\right)^{n}\right|_{\Delta X^{n}}$, with $Y$ a subbundle of $\left.\left(P^{*}\right)^{n}\right|_{\Delta X^{n}}$.

Definition 1.5. $\left(I^{*}, L^{*}, \varphi, P^{*}, M^{*}\right)$ is a well-posed valued differential system if the following conditions are satisfied:

(i) $\left(I^{*}, L^{*}, \varphi\right)$ is a non-degenerate valued differential system (with $\operatorname{dim} Y=(n+1) m+n)$ and $\varphi=L \omega$ for a smooth function $L$ on $X$;

(ii) there exists a subbundle $P^{*}$ of $I^{*}$ of rank $m$ and a subbundle $M^{*}$ of $L^{*}$ of rank $m+n$ as in (1.15);

(iii) $\pi^{*}{ }^{*} M^{*}=\operatorname{span}\left\{\pi^{*} \theta \mid \theta \in C^{\infty}\left(X, M^{*}\right)\right\}$ is completely integrable on $Y$, where $\pi "=\pi \circ i$, and $i$ once more denotes the inclusion mapping $Y \rightarrow Z$ and $\pi$ the projection $Z \rightarrow X$.

$C F=\left\{\theta^{\alpha}, d u^{s+j}, \pi_{j^{\prime}}^{i^{\prime}}, \pi_{j}^{i^{\prime \prime}} \mid 1 \leq \alpha \leq s, 1 \leq i^{\prime} \leq s_{l}, j^{\prime} \in L_{i^{\prime}}, s_{l+1} \leq i^{\prime \prime} \leq\right.$ $s, 1 \leq j \leq n\}$ for $T^{*} X$ with $L_{i^{\prime}} \subset\{k \in N, 1 \leq k \leq n\}$ :

(i)

$$
I^{*}=\operatorname{span}\left\{\theta^{\alpha} \mid 1 \leq \alpha \leq s\right\}
$$

(ii)

$$
L^{*}=\operatorname{span}\left\{\theta^{\alpha}, d u^{s+j} \mid 1 \leq \alpha \leq s, 1 \leq j \leq n\right\} ;
$$

(iii) $T^{*} X=L^{*} \oplus R^{*}\left(\oplus\right.$ denotes a direct sum) with $R^{*}=\operatorname{span}\left\{\pi_{j^{\prime}}^{i^{\prime}}, \pi_{j}^{i^{\prime \prime}} \mid 1 \leq\right.$ (iv) $\left.i^{\prime} \leq s_{l}, j^{\prime} \in L_{i^{\prime}}, s_{l+1} \leq i^{\prime \prime} \leq s, 1 \leq j \leq n\right\}$

$$
d \theta_{j^{\prime}}^{i^{\prime}} \equiv 0 \bmod I, \text { for } j^{\prime \prime} \notin L_{i^{\prime}}\left\{\theta_{j^{\prime \prime}}^{i^{\prime}}=\theta^{i^{\prime}} \wedge \omega_{j^{\prime \prime}}\right\} ;
$$

(v)

$$
d \theta_{j^{\prime}}^{i^{\prime}} \equiv \pi_{j^{\prime}}^{i^{\prime}} \wedge \omega \bmod I, \text { for } j^{\prime} \in L_{i^{\prime}}
$$

$$
d \theta_{j}^{i \prime \prime} \equiv \pi_{j}^{i \prime \prime} \wedge \omega \bmod I, \text { when } 1 \leq j \leq n ;
$$

(vii) $\pi_{j^{\prime}}^{i^{\prime}}, \pi_{j}^{i \prime}$ are linearly independent $\bmod L$. 
In [29] we presented a set of boundary conditions for different types of Well-posed valued differential systems. Solutions of the Cartan system with these boundary conditions have null first variations i.e. they are soutions of the Euler-Lagrange system.

\section{Generalized Lagrange Problem.}

Let $X=J^{1}\left(R^{n}, R^{m}\right)$ (the 1 jet manifold), with the canonical system $I^{*}$ defined on $X$ (i.e. $I^{*}=\operatorname{span}\left\{\theta^{\alpha}=d y^{\alpha}-y_{x^{i}}^{\alpha} d x^{i}\right\}$ ). Let $\varphi=L \omega$ with $\omega=d x^{1} \wedge \ldots \wedge d x^{n}$. We choose $x^{1}, \ldots, x^{n}$ to be coordinates for $R^{n}$, and $y^{1}, \ldots, y^{m}$ coordinates for $R^{m}$.

Definition 2.1. Let $f$ be a solution to the canonical differential system $I^{*}$, with the independence condition given by $L^{*}=\operatorname{span}\left\{I^{*}, d x^{1}, \ldots, d x^{n}\right\}$ and $k \in N$. The family $F\left(x, t_{1}, \ldots, t_{k}\right)$ of integral manifolds of $\left(I^{*}, L^{*}\right)$ is a $k$-parameter variation of $f$ if:

(i) $F\left(x, t_{1}, \ldots, t_{k}\right)$ is smooth with $\left(t_{1}, \ldots, t_{k}\right) \in\left[0, \epsilon_{1}\right] \times \ldots \times\left[0, \epsilon_{k}\right]$, for $\epsilon_{i}>0,1 \leq i \leq k$

(ii) $F_{\left(t_{1}, \ldots, t_{k}\right)} \doteq \bar{F}\left(x, t_{1}, \ldots, t_{k}\right) \in V\left(I^{*}, L^{*}\right)$ for all $\left(t_{1}, \ldots, t_{k}\right) \in\left[0, \epsilon_{1}\right] \times$ $\ldots \times\left[0, \epsilon_{k}\right]$,

(iii) $F(x, 0)=f(x)$ for all $x \in N, N \subset R$.

$F_{*}\left(\frac{\partial}{\partial t_{i}}\right)$ is an infinitesimal variation of $F$.

We will consider variations satisfying the condition $\pi^{\prime \prime}(F(x, t))=\pi^{\prime \prime}(f(x))$ for all $x \in \partial N$ and $t \in[0, \epsilon] \quad\left(\pi^{\prime \prime}\right.$ is the projection $\left.J^{1}\left(R^{n}, R^{m}\right) \rightarrow R^{n}\right)$.

Without loss of generality we can choose $v$ so that $v\lrcorner d x^{i}=0$, thus replacing a one parameter variation of $f$ by another that has the same first and second variation while satisfying:

for all $t \in[0, \epsilon])$.

$$
\pi^{\prime \prime}(F(x, t))_{N}=i d_{N}
$$

The framework of this Lagrange problem subjected to constrains represents a very important set of problems for the study of Calculus of Variations.

\section{Inverse problem for calculus of variations.}

3.1. Two Examples. In 1887, Helmholtz solved the following problem:

Example 1. Given $P_{i}=P_{i}\left(x, u^{j}, u_{x}^{j}, u_{x x}^{j}\right)$. Is there a Lagrangian $L\left(x, u^{j}, u_{x}^{j}\right)$ such that $E_{i}(L)=\partial L / \partial u^{i}-D_{x} \partial L / \partial u_{x}^{i}=P_{i}$ where $D_{x}=$ $\partial / \partial x+u_{x}^{i} \partial / \partial u^{i}+u_{x x}^{i} \partial / \partial u_{x}^{i}$ ? He found the necessary conditions for $P_{i}$ : 
(i)

$$
\partial P_{i} / \partial u_{x x}^{j}=\partial P_{j} / \partial u_{x x}^{i}
$$

$$
\partial P_{i} / \partial u^{j}=\partial P_{j} / \partial u^{i}-D_{x} \partial P_{j} / \partial u_{x}^{i}+D_{x x} \partial P_{j} / \partial u_{x x}^{i} .
$$

This problem led to the following studies [4].

(i) - the derivation and analysis of Helmholtz conditions as necessary and (locally) sufficient conditions for a differential operator to coincide with the Euler-Lagrange operator for some Lagrangian;

ii) - the characterization of the obstructions to the existence of global variational principles for different operators defined on manifolds;

iii) - the invariant inverse problem for different operators with symmetry; and

(iv) - the variational multiplier problem wherein variational principles are found, not for a given differential operator, but rather for the differential equations determined by that operator.

That is: find a matrix $B=\left[B_{i}^{j}\right]$ such that $B_{i}^{j} P_{j}=E_{i}(L)$ for some $L$, with $B$ being non-singular.

Let $E \rightarrow M$ be a fibered manifold. $J^{\infty}(E)$ is the infinite jet of $E$.

Let

and

$$
\begin{gathered}
\theta^{i}=d u^{i}-u_{x}^{i} d x \\
\theta_{x}^{i}=d u_{x}^{i}-u_{x x}^{i} d x
\end{gathered}
$$

$$
\begin{gathered}
\Omega_{P}=P_{i} \theta^{i} \wedge d x+1 / 2\left[\partial P_{i} / \partial u_{x}^{i}-D_{x} \partial P_{i} / \partial u_{x x}^{i}\right] \theta^{i} \wedge \theta^{j} \\
+1 / 2\left[\partial P_{i} / \partial u_{x x}^{i}+\partial P_{j} / \partial u_{x x}^{i}\right] \theta^{i} \wedge \theta_{x}^{j} .
\end{gathered}
$$

If $P$ satisfies the Helmholtz conditions, then $d \Omega_{P}=0$. If $\Omega_{P}$ is exact (equivalently if $H^{n+1}(E) \mathrm{n}+1$ de Rham cohomology group of $\mathrm{E}$ is trivial), then $P_{i}$ is globally variational.

If $\theta_{L}=L d x+\partial L / \partial u_{x}^{i} \theta^{i}$, then $d \theta_{L}=\Omega_{P}$.

In 1913, Volterra [50] showed that if $L=\int_{N} u^{i} P_{i}\left(x, t u^{j}, t u_{x}^{j}, t u_{x x}^{j}\right) d t$ where $N=[0,1]$, then:

$$
E_{i}(L)=P_{i}
$$

We have a global solution to the inverse problem in the case of one independent variable and to equations $P_{i}=0$ of second order.

Vaingberg 1964 [49] generalized this result, however this Lagrangian is usually of much higher order than necessary. 
From [5] one can derive the following theorem:

Theorem 3.1. Let $\Delta$ be a differential operator of order $2 k$

$$
\Delta=P_{\beta}\left(x^{i}, u^{j}, u_{1}^{j}, \ldots, u_{2 k}^{j}\right) \theta^{\beta} \wedge \omega .
$$

Then $\Delta$ is the Euler-Lagrange operator of a $k-t h$ order Lagrangian $L=$ $L\left(x^{i}, u^{j}, u_{1}^{j}, \ldots, u_{k}^{j}\right)$, if and only if $\Delta$ satifies the higher order Helmholtz conditions, and the functions

$$
p_{\beta}(t)=P_{\beta}\left(x^{i}, u^{j}, u_{1}^{j}, \ldots, u_{k}^{j}, t u_{k+1}^{j} \ldots, t^{k} u_{2 k}^{j}\right)
$$

are polynomials in $t$ of degree less or equal to $k$.

The functions $u_{k}^{j}$ denote all possible $k^{t h}$-order derivatives of $u^{j}, 1 \leq$ $\beta, j \leq m$ and $1 \leq i \leq n, \theta^{\beta}=d y^{\beta}-y_{x^{i}}^{\beta} d x^{i}$ and $\omega=d x^{1} \wedge \ldots \wedge d x^{n}$.

Example 2. Let $T=T\left(x, y, z, u, u_{x}, u_{y}, u_{z}, u_{x x}, u_{x y}, \ldots, u_{z z}\right)$ be a second order operator. We assume that $T$ is a smooth function.

Let $L=L\left(x, y, z, u, u_{x}, u_{y}, u_{z}\right)$ be a first-order operator with $L$ being a smooth function.

where

$$
E[L]=\partial L / \partial u-D_{x} \partial L / \partial u_{x}-D_{y} \partial L / \partial u_{y}-D_{z} \partial L / \partial u_{z}
$$

$$
D_{x}=\partial / \partial x+u_{x} \partial / \partial u+u_{x x} \partial / \partial u_{x}+u_{x y} \partial / \partial u_{y}+\ldots
$$

Let $v$ be a lift to the momentum space of an infinitesimal variation $F_{*}(\partial / \partial t)$ of $f=\pi o g$, where $g$ is a solution of $\left(C(\Psi), \pi^{*} \omega\right)$. The Liederivative of $\psi=\pi^{*} \varphi+\left(\pi^{j} o i^{\prime}\right)^{*}\left[i^{*}(\chi)\right] \wedge \pi^{*} \omega_{j}$ along $v$ is:

$$
\begin{gathered}
v\lrcorner d \psi+d(v\lrcorner \psi)=E[L](u) v^{1} \pi^{*}(d x \wedge d y \wedge d z) \\
+d\left(\partial L / \partial u_{x} v^{1} \pi^{*}(d y \wedge d z)-\partial L / \partial u_{y} v^{1} \pi^{*}(d x \wedge d z)\right. \\
\left.+\partial L / \partial u_{z} v^{1} \pi^{*}(d x \wedge d y)\right)
\end{gathered}
$$

Suppose that for every vector $v$ with $\pi_{*} v \in T_{f} V\left(I^{*}, L^{*}, \varphi,[h]\right)$ we have a vector $w$ with $\pi_{*} w \in T_{f} V\left(I^{*}, L^{*}, \varphi,[h]\right)$ such that

$$
\begin{gathered}
v\lrcorner d \psi+d(v\lrcorner \psi)=T[u] v^{1} \pi^{*}(d x \wedge d y \wedge d z) \\
+d\left(\partial L / \partial u_{x} w^{1} \pi^{*}(d y \wedge d z)-\partial L / \partial u_{y} w^{1} \pi^{*}(d x \wedge d z)\right. \\
\left.+\partial L / \partial u_{z} w^{1} \pi^{*}(d x \wedge d y)\right) .
\end{gathered}
$$

Then we have $T[u]=E[L](u)$.

If we identify $e_{1}$ with $\pi^{*}(d y \wedge d z), e_{2}$ with $\pi^{*}(d z \wedge d y)$ and $e_{3}$ with $\pi^{*}(d x \wedge d y)$ at each point of the integral manifold of $\left(C(\Psi), \pi^{*} \omega\right)$, we can write:

$d\left(\partial L / \partial u_{x} v^{1} \pi^{*}(d y \wedge d z)-\partial L / \partial u_{y} v^{1} \pi^{*}(d x \wedge d z)+\partial L / \partial u_{z} v^{1} \pi^{*}(d x \wedge d y)\right)$ 
where

$$
=\operatorname{Div} V[u] \pi^{*}(d x \wedge d y \wedge d z),
$$

$$
V[u]=\partial L / \partial u_{x} v^{1} e_{1}+\partial L / \partial u_{y} v^{1} e_{2}+\partial L / \partial u_{z} v^{1} e_{3} .
$$

We can conclude that

$$
v\lrcorner d \psi+d(v\lrcorner \psi)=(E[L](u) v+\operatorname{Div} V[u]) \pi^{*}(d x \wedge d y \wedge d z) .
$$

We have

$$
E[L](u)=0 \quad \text { if } \quad L[u]=\operatorname{Div} W[u] .
$$

Suppose $T[u]=E[L](u)$. Then

$$
E(E[L](u) v+\operatorname{Div} V[u])=E[T(u) v] .
$$

Let

$$
\psi^{\prime}=\pi^{*} T \omega+\left(\pi^{j} o i^{\prime}\right)^{*}[i *(\chi)] \pi^{*} \omega_{j}
$$

If we define

$\left.\left.H(T)[v] \pi^{*}(d x \wedge d y \wedge d z)=v\right\lrcorner d \psi^{\prime}+d(v\lrcorner \psi^{\prime}\right)-E[T(u) v] \pi^{*}(d x \wedge d y \wedge d z)$, then $H(T)=0$ if $T[u]$ is Euler-Lagrange. Helmholtz equations are:

(i) $\partial T / \partial u_{x}=D_{x} \partial T / \partial u_{x x}+1 / 2 D_{y} \partial T / \partial u_{x y}+1 / 2 D_{z} \partial T / \partial u_{x z}$,

(ii) $\partial T / \partial u_{y}=D_{y} \partial T / \partial u_{x x}+1 / 2 D_{x} \partial T / \partial u_{y x}+1 / 2 D_{z} \partial T / \partial u_{y z}$,

(iii) $\partial T / \partial u_{z}=D_{z} \partial T / \partial u_{x x}+1 / 2 D_{x} \partial T / \partial u_{z x}+1 / 2 D_{y} \partial T / \partial u z z$.

We have a sequence of spaces

$$
0 \rightarrow R \rightarrow F[u] \stackrel{\text { Grad }}{\longrightarrow} V(u) \stackrel{\text { Curl }}{\longrightarrow} V(u) \stackrel{\text { Div }}{\longrightarrow} F(u) \stackrel{E}{\longrightarrow} F(u) \stackrel{H}{\longrightarrow} V(u)
$$

that is a cochain complex, the Euler-Lagrange complex, where $F[u]$ is the set of smooth functions $F\left(x, y, z, u, u_{x}, u_{y}, u_{z}, u_{x x}, u_{x y}, \ldots, u_{z z}\right), V[u]$ is the set of vector fields defined in $R^{n}$ with $F[u]$ coeficients. This complex is exact and thus the inverse problem is solved in this second example.

3.2. Variational Bicomplex. Let us introduce now a very important tool for a globalization of the inverse problem [40].

Definition 3.1. A p form $\omega$ on $J^{\infty}(E)$ is said to be of type $(r, s)$, where $r+s=p$, if at each point $x$ of $J^{\infty}(E)$,

$$
\omega\left(X_{1}, X_{2}, \ldots, X_{p}\right)=0,
$$

whenever either

(i) more than $s$ of the vectors $X_{1}, X_{2}, \ldots, X_{p}$ are $\pi_{M}^{\infty}$ vertical, or

(ii) more than $r$ of the vectors $X_{1}, X_{2}, \ldots, X_{p}$ annihilate all contact one forms. 
Note: $\Omega^{r, s}$ denotes the space of type $(r, s)$ forms on $J^{\infty}(E)$.

(i) $\pi: E \rightarrow M$ is a fiber bundle.

(ii) In some cases we can assume that there exists a transformation group $G$ acting on $E$.

(iii) There exists a set of differential equations on sections of $E$.

$$
\begin{gathered}
d=d_{H}+d_{V} \\
d_{H}: \Omega^{r, s}\left(J^{\infty}(E)\right) \rightarrow \Omega^{r+1, s}\left(J^{\infty}(E)\right), \\
d_{V}: \Omega^{r, s}\left(J^{\infty}(E)\right) \rightarrow \Omega^{r, s+1}\left(J^{\infty}(E)\right) \\
d_{H}^{2}=0, d_{H} d_{V}=-d_{V} d_{H}, d_{V}^{2}=0
\end{gathered}
$$

In local coordinates

$$
\begin{gathered}
d_{H} f=\left[\partial f / \partial x^{i}+u \alpha_{i} \partial f / \partial u^{\alpha}+u_{i j}^{\alpha} \partial f / \partial u_{j}^{\alpha}+\ldots\right] d x^{i} \\
d_{V} f=\partial f / \partial u^{\alpha} \theta^{\alpha}+\partial f / \partial u_{i}^{\alpha} \theta_{i}^{\alpha}+\ldots
\end{gathered}
$$

I is locally given by:

$$
\begin{gathered}
\left.\left.I=\Omega^{r, s}\left(J^{\infty}(E)\right)\right) \rightarrow \Omega^{r, s}\left(J^{\infty}(E)\right)\right) \\
\left.I(\omega)=\frac{1}{s} \theta^{\alpha} \wedge\left[\left(\partial / \partial u^{\alpha}\right\lrcorner \omega\right)-D_{i}\left(\left(\partial / \partial u_{i}^{\alpha}\right\lrcorner \omega\right)+D_{i j}\left(\left(\partial / \partial u_{i j}^{\alpha}\right\lrcorner \omega\right)-\ldots\right]
\end{gathered}
$$

Definition 3.2. The sequences of spaces

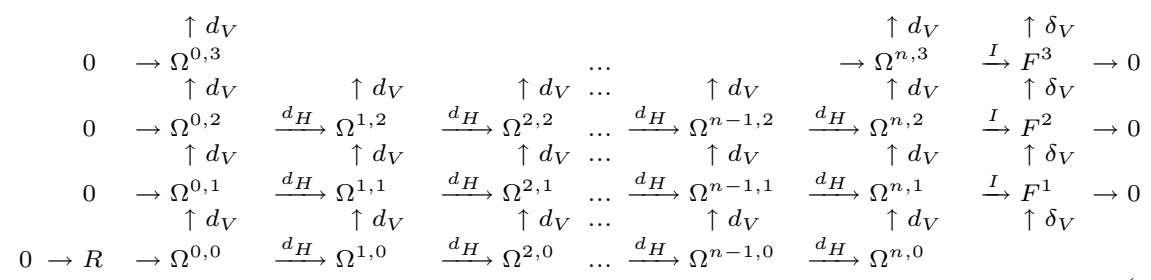

is the Variational Bicomplex.

Therefore the generalization of (3.15) is:

$$
0 \rightarrow R \rightarrow \Omega^{0,0} \stackrel{d_{H}}{\longrightarrow} \Omega^{1,0} \stackrel{d_{H}}{\longrightarrow} \Omega^{2,0} \ldots \stackrel{d_{H}}{\longrightarrow} \Omega^{n-1,0} \stackrel{d_{H}}{\longrightarrow} \Omega^{n, 0} \stackrel{E}{\longrightarrow} F^{1} \stackrel{\delta_{H}}{\longrightarrow} F^{2} \stackrel{\delta_{H}}{\longrightarrow} F^{3} \ldots
$$




\section{G.L. Problem with non-holonomic constraints}

4.1. G.L. Problem with non-holonomic constraints. Let us recall from [26] the Generalized Lagrange problem with non-holonomic constraints for $n>1, m=1$.

Let us assume $g^{\rho}\left(x^{i}, u, u_{x^{j}}\right)=0$, with $\operatorname{rank}\left[\partial g^{\rho} / \partial u_{x^{j}}\right]=n-l$, $g^{\rho}\left(x^{i}, u, u_{x^{j}}\right)$ smooth functions with $1 \leq j \leq n, 1 \leq \rho \leq n-l$ and $l \geq 0$ $\left(I^{*}, L^{*}, \varphi, I^{*}, L^{*}\right)$ is a well-posed valued differential system, where: $I^{*}=$ span $\{\theta\}$, and $L^{*}=\operatorname{span}\left\{\theta, d x^{i} \mid 1 \leq i \leq n\right\}$

$$
\theta=\left\{d u-u_{x^{i}} d x^{i} \mid 1 \leq i \leq n\right\} .
$$

In this setting we have:

$$
\begin{gathered}
d \theta_{\mu}=-d u_{x^{\mu}} \wedge \omega \\
d \theta_{\rho}=-\left(A_{\rho}^{\mu} d u_{x^{\mu}} \wedge \omega-B_{\rho} \theta \wedge \omega\right) \\
A_{\rho}^{\mu}=-a_{\sigma}^{\rho} g_{u_{x^{\mu}}}^{\sigma} \\
B_{\rho}=-a_{\sigma}^{\rho} g_{u}^{\sigma} \\
L^{\mu}=\left(\partial / \partial d u_{x^{\mu}}-A_{\rho}^{\mu} \partial / \partial u_{x^{\rho}}\right) L, \\
L^{\mu \nu}=\left(\partial / \partial d u_{x^{\mu}}-A_{\rho}^{\mu} \partial / \partial u_{x^{\rho}}\right) L^{\nu},
\end{gathered}
$$

and

$$
\begin{gathered}
{\left[a_{\sigma}^{\rho}\right]=\left[g_{u_{\rho}}^{\sigma}\right]^{-1} \text { with } 1 \leq \rho, \sigma, \leq n-l \text { and } n-l+1 \leq \mu, \nu \leq n} \\
\Psi \equiv\left(L^{\mu}-\lambda^{\mu}-\lambda^{\rho} A_{\rho}^{\mu}\right) \pi^{*}\left(d u_{x^{\mu}} \wedge \omega\right)+d \lambda^{\mu} \wedge \pi^{*}\left(\theta_{\mu}\right)+d \lambda^{\rho} \wedge \pi^{*}\left(\theta_{\rho}\right) \\
+\left(L_{u}-\lambda^{\rho} B_{\rho}+L_{u_{x} \rho} B_{\rho}\right) \pi^{*}(\theta \wedge \omega)
\end{gathered}
$$

The Cartan system is:

$$
\begin{gathered}
\pi^{*} \theta_{\alpha} \quad(1 \leq \alpha \leq n) \\
\left(L^{\mu}-\lambda^{\mu}-\lambda^{\rho} A_{\rho}^{\mu}\right) \pi^{*} \omega \quad(n-l+1 \leq \mu \leq n) \\
\left(-d \lambda^{\rho} \wedge \pi^{*} \omega_{\rho}-d \lambda^{\mu} \wedge \pi^{*} \omega_{\mu}\right)+\left(L_{u}+\lambda^{\rho} B_{\rho}-L_{u_{x} \rho} B_{\rho}\right) \wedge \pi^{*} \omega
\end{gathered}
$$

Let us assume $g^{\rho} / \partial u_{x^{\mu}}=0$ for all $n-l+1 \leq \mu \leq n$ and all $1 \leq \rho \leq n-l$. Then the Euler-Lagrange equation is: 


$$
E[L]=\partial L / \partial u+\partial L / \partial u_{x^{\sigma}} B_{\sigma}-D_{x^{\mu}} \partial L / \partial u_{x^{\mu}}+\lambda^{\sigma} B_{\sigma}-\lambda_{x^{\rho}}^{\rho}
$$

Proposition 4.1. Let $\left(I^{*}, L^{*}\right)$ be a locally embeddable differential system defined in $X=\left.J^{1}\left(R^{n}, R\right)\right|_{g^{\rho}\left(x^{i}, u, u_{x^{j}}\right)=0}, \operatorname{rank}\left[\partial g^{\rho} / \partial u_{x^{j}}\right]=n-l$, $g^{\rho}\left(x^{i}, u, u_{x^{j}}\right)$ smooth functions, $1 \leq i, j \leq n, 1 \leq \rho \leq n-l, l \geq 0$, and $g^{\rho} / \partial u_{x^{\mu}}=0$ for all $n-l+1 \leq \mu \leq n$ and all $1 \leq \rho \leq n-l$, where $I^{*}=$ $\operatorname{span}\{\theta\}$,

$L^{*}=\operatorname{span}\left\{\theta, d x^{i} \mid 1 \leq i \leq n\right\}$,

$$
\theta=d u-u_{x^{j}} d x^{j} \quad 1 \leq j \leq n .
$$

Let $Q\left(x^{i}, u, u_{x^{\mu}}, u_{x^{\mu} x^{\nu}}, \lambda^{\rho}, \lambda_{x^{i}}^{\rho}\right)$ with $n-l+1 \leq \mu, \nu \leq n, 1 \leq \sigma, \rho \leq n-l$ and $1 \leq i \leq n$, with $Q\left(x^{i}, u, u_{x^{\mu}}, t u_{x^{\mu} x^{\nu}}, \lambda^{\rho}, \lambda_{x^{i}}^{\rho}\right)$ being a polynomial in $t$ of degree less or equal to 1 , and

$$
P=Q+\lambda_{x^{\rho}}^{\rho}-\lambda^{\rho} B_{\rho} .
$$

And furthermore, let us assume that $P$ satisfies the Helmholtz conditions and does not depend on $\lambda_{\rho}$ and $\lambda_{x^{i}}^{\rho}$ coordinates, then $Q$ is locally a EulerLagrange operator for a Lagrangian $L\left(x^{i}, u, u_{x^{\mu}}\right)$.

Proof: In this case the Helmholtz condition is:

$$
\partial P / \partial u_{x^{\mu}}=D_{x^{\mu}} \partial P / \partial u_{x^{\mu} x^{\mu}}+1 / 2 D_{x^{\nu}} \partial P / \partial u_{x^{\mu} x^{\nu}},
$$

with $n-l+1 \leq \mu, \nu \leq n$.

From Theorem 3.1 we know that a function $F\left(x^{i}, u, u_{x^{\mu}}\right)$ can be found that does not depend on $u_{x x}^{\nu}$, such that $E[F]=\partial F / \partial u-D_{x}^{i} \partial F / \partial u_{x}^{i}$.

In addition, if in the domain of $P$ the sequence of spaces is exact:

$$
\Omega^{n, 0} \stackrel{E}{\longrightarrow} F^{1} \stackrel{H}{\longrightarrow} 0
$$

then we have a global solution for the inverse problem.

Example 3. Let $X=\left.J^{1}\left(R^{n}, R\right)\right|_{g^{\rho}\left(x^{i}, u, u_{x^{j}}\right)=0}, \operatorname{rank}\left[\partial g^{\rho} / \partial u_{x^{j}}\right]=n-l$, $g^{\rho}\left(x^{i}, u, u_{x^{j}}\right)$ smooth functions, $1 \leq i, j \leq n, 1 \leq \rho \leq n-l, l \geq 0$, and $g^{\rho} / \partial u_{x^{\mu}}=0$ for all $n-l+1 \leq \mu \leq n$ and all $1 \leq \rho \leq n-l$, where $I^{*}=\operatorname{span}\{\theta\}, L^{*}=\operatorname{span}\left\{\theta, d x^{i} \mid 1 \leq i \leq n\right\}$ and $Q\left(x^{i}, u, u_{x^{\mu}}, u_{x^{\mu} x^{\nu}}\right)=$ $2 u_{x^{\sigma}}\left(x^{i}, u\right) B_{\sigma}-\sum_{\mu} 2 u_{x^{\mu} x^{\mu}}+\lambda^{\sigma} B_{\sigma}-\lambda_{x^{\rho}}^{\rho}$. Q satisfies Helmhotz equations and is globally a Euler-Lagrange operator with $L=\sum_{\mu}\left(u_{x^{\mu}}\right)^{2}+\sum_{\sigma}\left(u_{x^{\sigma}}\left(x^{i}, u\right)\right)^{2}$. 


\section{References}

[1] I. M. Anderson. Natural variational principles on Riemannian manifolds Ann. of Math., Volume 120, pp 329-370, 1984.

[2] I. M. Anderson. Introduction to the variational bicomplex Contemporary Mathematics, Volume 132, pp 51-73, 1992.

[3] I. M. Anderson. On the existence of global variational principles Amer. J. Math., Volume 102, pp 781-868, 1980.

[4] I. M. Anderson and G. Thompson. The inverse problem of the calculus of variations for ordinary differential equations Memoirs Amer. Math. Soc., Volume 98, pp vi+110, 1992.

[5] I. M. Anderson. The Variational Bicomplex http : //bib.tiera.ru/b/37499, (book draft, free web version, Utah, 2004), pdf.

[6] R. Bryant, S. S. Chern, R. Gardner and P. Griffiths. Essays on exterior differential systems Springer-Verlag, New York, 1990.

[7] C. Caratheodory. Variationsrechnung bei mehrfachen Integralen Acta Szeged 4, 1929.

[8] E. Cartan. Les systémes differentielles exterieurs et leurs applicationsgéométriques Herman, Paris, 1945.

[9] P. Dedecker. Calcul des variations, formes differentielles et champs geodé siques Colloques. Internat. du C.N.R.S., Strasbourg, 1953.

[10] P. Dedecker. Calcul des variations et topologie algebraique Mem. Soc. Roy. Sc. Liége, 4-e série, XIX, fase I, 1957.

[11] P. Dedecker. On the generalization of sympletic geometry to muliple integrals in the calculus of variations Lecture Notes in Math. Springer, Vol. 570, Berlin and New York, 1977.

[12] Th. De Donder. Téorie invariantive du calcul de variations Gautier-Villars, Paris, 1935.

[13] D. G. B. Edelen. The invariance group for Hamiltonian systems of partial differential equationss Arch.Rational Mech. Anal., Volume 5 pp 95-176, 1961.

[14] D. G. B. Edelen. Nonlocal variations and local invariance of fields American Elsvier, New York, 1969.

[15] H. I. Eliasson Variational integrals in fiber bundles Proc. Sympos. Pure Math. Amer. Math. Soc., Volume 16, Providence, RI, pp 67-89 1970.

[16] P. L. Garcia, The Poincaré-Cartan invariant in the calculus of variations Sympos. Math., Volume 14, pp 219-227, 1974.

[17] P. L. Garcia, Gauge algebras, curvature and symplectic structure J. Differential Geometry, Volume 12, pp 209-246, 1977.

[18] P. L. Garcia, Critical principal connections and gauge invariance Rep. Math. Phys, Volume 13, pp 337-344, 1978.

[19] P. L. Garcia, Tangent structure of Yang-Mills equations and Hodge Theory Lecture Notes in Math. Springer, Volume 775, Berlin and New York, 1980.

[20] P. L. Garcia, A. Pérez-Rendón. Symplectic approach to the theory of quantized fields I Comm. Math Phys., Volume 13, pp 24-44, 1969.

[21] P. L. Garcia, A. Pérez-Rendón. Symplectic approch to the theory of quantized fields. II Arch. Rational Mech. Anal, Volume 43, pp 101-124, 1971.

[22] P. L. Garcia, A. Pérez-Rendón. Reducibility of the symplectic structure of minimal interactions Lecture Notes in Math. Springer, Volume 676, Berlin and New York, pp 409-433, 1978.

[23] H. Goldschmidt, S. Sternberg, The Hamilton-Cartan formalism in the calculus of variations Ann. Inst. Fourier, Volume 23, Grenoble, pp 203-267, 1973. 
[24] P. Griffiths. Exterior differential systems and the calculus of variations Birkäuser, Boston, Basel, Stuttgart, 1983.

[25] C. Günther. The plysymplectic Hamiltonian formalism in the field theory and calculus of variations I: The local case J. Differential Geometry, Volume 25, pp 23-53, 1987.

[26] P. G. Henriques. Calculus of variations in the context of exterior differential systems Differential Geometry and its Applications (North-Holland), Volume 3, pp 331-372, 1993.

[27] P. G. Henriques. Well-posed variational problem with mixed endpoint conditions Differential Geometry and its Applications (North-Holland), Volume 3, pp 373393, 1993.

[28] P. G. Henriques. The Noether theorem and the reduction procedure for the variational calculus in the context of differential systems C. R. Acad. Sci. Paris Sér. I Math., Volume 317, pp 987-992, 1993.

[29] P. G. Henriques. The inverse problem of variational calculus and the problem of mixed endpoint conditions São Paulo Journal of Mathematical Science, Volume 2, pp 239-262, 2008.

[30] R. Hermann. Differential geometry and the calculus of varitions Academic Press, New York, 1968.

[31] J. Kijowski, W. M. Tulczyjew. A symplectic framework for field theories Lecture Notes in Math. Springer, Volume 107, Berlin and New York, 1979.

[32] D. Krupka. Lagrange theory in fibered manifolds Rep. Math. Phys, Volume 2, pp 121-133, 1970.

[33] D. Krupka. A geometric theory of ordinary first order variational problems in fibered manifolds I: Critical sections J. Math Anal. Appl., Volume 49, pp 180206, 1975.

[34] D. Krupka. A geometric theory of ordinary first order variational problems in fibered manifolds II: Invariance J. Math Anal. Appl., Volume 49, pp 469-476, 1975.

[35] T. Lepage. Sur les champs geodésiques des integrals multiples Bull. Acad. Roy. Belg, CI. Sc., 5.éme série 22, 1936.

[36] T. Lepage. Sur les champs geodésiques des integrals multiples Bull. Acad. Roy. Belg, CI. Sc., 5.éme série 27, 1941.

[37] T. Lepage. Champs stationnaires, champs geodésiques et formes integrables I, II Bull. Acad. Roy. Belg, CI. Sc., 5.éme série 28, 1942.

[38] A. Liesen. Feldtheorie in der Variatonrechnung mehfacher Integrale I Math Annalen, Volume 171, pp 194-218 1967.

[39] A. Liesen. Feldtheorie in der Variatonrechnung mehfacher Integrale II Math Annalen, Volume 171, pp 273-292 1967.

[40] Waldyr M. Oliva and Gláucio Terra. An Inverse Problem on Vakonomic Mechanics São Paulo Journal of Mathematical Science, Volume 5, 1, pp 23-35, 2011.

[41] P. J. Olver. Euler operators and conservation laws of BBM equation Math. Proc. Cam. Phil. Soc., Volume 85, pp 143-160, 1979.

[42] P. J. Olver. Applications of Lie groups to differential equations Springer, New York, 1986.

[43] R. Ouzilou. Expression symplectic des problems variationnels Sympos. Math , Volume 14, pp 85-98, 1972.

[44] H. Rund. The Hamilton-Jacobi theory in the calculus of variations Van Nostrand, Princeton, NJ, 1966.

[45] F. Takens. Symmetries, conservation laws and variational principles Lectures Notes in Mathematics, Springer, Volume 597, pp 581-603, New York, 1977. 
[46] F. Takens. A global version of the inverse problem to the calculus of variations J. Differential Geometry, Volume 14, pp 543-562, 1979.

[47] W. M. Tulczyjew. The Euler-Lagrange resolution Lectures Notes in Mathematics, Springer, Volume 836, pp 22-48 S, New York, 1980.

[48] W. M. Tulczyjew. Cohomology of the Lagrange complex Ann. Scuola. Norm. Sup. Pisa, pp 217-227, 1988.

[49] M. M. Vainberg Variational Methods for tht study of Nonlinear Operators Holden-Day, S. Francisco, 1964.

[50] A. M. Vinagradov. The C-spectral sequence, Lagrangian formalism and conservation laws I, II J. Math Anal. Appl, Volume 100, pp 1-129, 1984.

[51] A. M. Vinagradov. Symmetries and conservation laws of partial differential equations: basic notions and results Acta Appl. Math., Volume 15, pp 3-22, 1989.

[52] A. M. Vinagradov. Scalar differential invariants, diffiieties and characteristic classes Mechanics, Analysis and Geometry: 200 Years after Lagrange, M. Francavglia(ed), Elsevier, Amsterdam, pp 379-416, 1991.

[53] V. Volterra. Leçons sur les Fonctions de Lignes Gautherier-Villars, Paris, 1913.

[54] H. Weyl. Geodesic fields in the calculus of variations for multiple integrals Annals of Math., Volume 36, pp 607-629 1935. 